\title{
Biofiltração da água e tipos de substrato na larvicultura do pacamã
}

\author{
Marcelo Mattos Pedreira(1), Ronald Kennedy Luz ${ }^{(1)}$, José Cláudio Epaminondas dos Santos(2), \\ Edson Vieira Sampaio( ${ }^{(2)}$ e Rafael Sá Fortes Silva(1)
}

\begin{abstract}
(1)Universidade Federal dos Vales do Jequitinhonha e Mucuri, Departamento de Zootecnia, Laboratório de Aquicultura e Ecologia Aquática, Rua da Glória, no 187, Centro, CEP 39100-000 Diamantina, MG. E-mail: marcelo_ufvjm@hotmail.com, luzrk@yahoo.com, rafaelsfs89@hotmail.com (2)Companhia de Desenvolvimento dos Vales do São Francisco e do Parnaíba, Estação de Hidrobiologia e Piscicultura de Três Marias, Caixa Postal 11, CEP 39205-000 Três Marias, MG. E-mail: jose.claudio@codevasf.gov.br, alicedson@bol.com.br
\end{abstract}

Resumo - O objetivo deste trabalho foi avaliar o uso de diferentes biofiltros e substratos na qualidade da água e no desempenho de larvas de Lophiosilurus alexandri. Os tratamentos usados foram: sistema sem biofiltro (SB); biofiltro interno ao tanque com substrato de cascalho de diâmetro médio de 1,6 cm $(\mathrm{CMe})$; biofiltro interno ao tanque com cascalho de diâmetro médio de $2,1 \mathrm{~cm}(\mathrm{CMa})$; biofiltro interno ao tanque com substrato constituído de uma mistura de $70 \%$ de brita (diâmetro médio de 1,3 cm) e $30 \%$ de concha (diâmetro médio de $1,1 \mathrm{~cm})(\mathrm{CB})$; e sistema fechado (SF) com biofiltro externo aos tanques, com substrato de brita e concha. $\mathrm{O}$ experimento foi realizado em delineamento inteiramente casualizado, com cinco tratamentos e cinco repetições, durante 16 dias. Para íon amônio, nitrito, nitrato e ortofosfato, houve interação entre sistemas $\mathrm{x}$ dias de cultivo. Os sistemas SF, CMe e CB registraram menores concentrações de íon amônio e nitrito; o sistema SB, maiores concentrações de íon amônio e menores concentrações de nitrato; o SF, concentrações de ortofosfato superiores. A sobrevivência de L. alexandri foi inferior no SF. O crescimento não foi afetado pelos diferentes sistemas. Os biofiltros melhoraram a qualidade da água quanto aos compostos nitrogenados em função do tipo de substrato.

Termos para indexação: Artemia, Lophiosilurus alexandri, qualidade da água, resíduo orgânico, sistema de recirculação de água.

\section{Water biofilter system and biofilter media on pacamã larviculture}

\begin{abstract}
The objective of this work was to investigate the use of different biofilter systems and biofilter media on water quality and performance of Lophiosilurus alexandri larvae. The treatments used were: no biofilter system (SB); internal biofilter with $1.6 \mathrm{~cm}$ average diameter shingle media $(\mathrm{CMe})$; internal biofilter with $2.1 \mathrm{~cm}$ average diameter shingle media $(\mathrm{CMa})$; a mix of $70 \%$ of grit $(1.3 \mathrm{~cm}$ average diameter) and $30 \%$ of shell media (1.1 cm average diameter) (CB); and a closed recirculating system with external biofilter with $70 \%$ grit and 30\% shell media (SF). The experiment was carried out in a completely randomized design, with five treatments and five replicates, during 16 days. Interaction between the rearing systems $\mathrm{x}$ rearing days affected the ammonium ion, nitrite, nitrate and orthophosphate concentrations. The SF, CMe and CB systems showed lower ammonium and nitrite concentrations; the SB system, higher ammonium ion concentrations and lower nitrate concentrations; the SF system, higher orthophosphate concentrations. L. alexandri survival was worst in the SF system. Fish growth was not affected by the different systems used. The biofilters provide better water quality in terms of nitrogenous waste depending on the biofilter media.
\end{abstract}

Index terms: Artemia, Lophiosilurus alexandri, water quality, organic waste, recirculating aquaculture system.

\section{Introdução}

O Brasil possui $13,7 \%$ da água doce superficial do planeta (Tundisi, 2003) e clima favorável, o que o torna um país com características adequadas para o desenvolvimento da aquicultura. No entanto, essa atividade vem sendo considerada disseminadora de espécies exóticas (Fernandes et al., 2003), de enfermidades (Maximiano et al., 2005) e poluidora devido à descarga de matéria orgânica em cursos de água, que contribui para a eutrofização (Kubitza, 1998; Hussar et al., 2004). O desenvolvimento de sistemas de cultivo eficientes que melhorem o aproveitamento da água e mitiguem possíveis impactos é necessário. Com isso a utilização de sistemas fechados de circulação de água vem se tornando uma das alternativas para a aquicultura (Ozório et al., 2004; Colt et al., 2006; Gutierrez-Wing \& Malone, 2006). 
Em sistemas fechados de circulação de água, os biofiltros são utilizados para diminuir as concentrações de amônia por meio da sua oxidação a nitrato pelas bactérias nitrificantes (Hagopian \& Riley, 1998). No entanto, para que a nitrificação ocorra de maneira eficiente é necessário levar em conta: o tipo de substrato, concentração de oxigênio dissolvido, temperatura, $\mathrm{pH}$, alcalinidade, turbulência, salinidade da água (Chen et al., 2006), área superficial específica do substrato e homogeneidade do fluxo de água no biofiltro (Lekang \& Kleppe, 2000).

Com o intuito de buscar melhor processo de nitrificação, diferentes tipos de biofiltros e substratos foram avaliados (Lekang \& Kleppe, 2000; Rhida \& Cruz, 2001; Al-Hafedh et al., 2003; Asano et al., 2003; Lyssenko \& Wheaton, 2006). Porém, os diversos sistemas utilizados em aquicultura vêm apresentando diferentes desempenhos e características operacionais, o que torna difícil selecionar o melhor sistema e realizar estudos devido ao grande número de parâmetros que devem ser medidos e controlados (Colt et al., 2006).

Apesar da necessidade de desenvolver sistemas de biofiltração para uma aquicultura mais sustentável, trabalhos sobre larvicultura de peixes neotropicais que utilizam sistemas fechados de circulação de água são escassos no Brasil (Pedreira, 2003).

O pacamã, Lophiosilurus alexandri, pertencente à família Pseudopimelodidae (ordem Siluriformes), é nativo da bacia do rio São Francisco, Minas Gerais (Shibata, 2003), e tem sido considerado ameaçado de extinção (Lins et al., 1997). É um peixe carnívoro, com comportamento sedentário e preferência por ambientes lênticos (Travassos, 1959). Manifesta cuidado parental, libera seus ovos no substrato arenoso (Sato et al., 2003) e tem alto valor de mercado pela carne sem espinhos intramusculares e pelo sabor apreciado pelo consumidor. Por esses motivos, esforços têm sido direcionados para o desenvolvimento de sua larvicultura e alevinagem (López \& Sampaio, 2000; Tenório et al., 2006; Luz \& Santos, 2008; Pedreira et al., 2008; Santos \& Luz, 2009).

O objetivo deste trabalho foi avaliar o uso de diferentes tipos de biofiltros e substratos na larvicultura do pacamã.

\section{Material e Métodos}

O experimento foi realizado na Estação de Hidrobiologia e Piscicultura de Três Marias, da
Companhia de Desenvolvimento dos Vales do São Francisco e do Parnaíba (Codevasf), em Três Marias, MG, no mês de janeiro de 2007, durante 16 dias.

Larvas de pacamã $(15,5 \pm 0,5 \mathrm{~mm}$ e $27,1 \pm 2,9 \mathrm{mg}$, início da alimentação exógena) foram estocadas na densidade de 2,5 larvas $\mathrm{L}^{-1}$ em 25 tanques com $30 \mathrm{~L}$ de água e fotoperíodo de 10 horas de claridade e14 horas de escuridão.

O experimento foi conduzido em delineamento inteiramente ao acaso, com cinco tratamentos e cinco repetições. Os tratamentos consistiram nos seguintes sistemas de biofiltração e substratos: biofiltro interno ao tanque (submerso) com substrato de cascalho de diâmetro médio de 1,6 cm (cascalho menor) (CMe); biofiltro interno ao tanque com substrato de cascalho de diâmetro médio de 2,1 cm (cascalho maior) (CMa); biofiltro interno ao tanque com substrato constituído de uma mistura de $70 \%$ de brita (diâmetro médio de $1,3 \mathrm{~cm}$ ) e $30 \%$ de concha (diâmetro médio de $1,1 \mathrm{~cm})(\mathrm{CB})$; biofiltro externo aos tanques, montado em sistema fechado de circulação de água com uma mistura de $70 \%$ de brita (diâmetro médio de 1,37 cm) e $30 \%$ de concha (diâmetro médio de 1,06 cm) (SF). Como controle foram utilizados tanques com aeração artificial por meio de pedra porosa, sem biofiltro (SB).

Os biofiltros internos aos tanques eram constituídos de um recipiente de $2 \mathrm{~L}(12 \times 18 \times 11 \mathrm{~cm})$ totalmente preenchido com os diferentes substratos. Cada biofiltro continha uma torre de "air lift" de PVC de $2 \mathrm{~cm}$ de diâmetro que emergia $5 \mathrm{~cm}$ acima do nível da água. Para o sistema fechado, os cinco tanques foram interligados por um sistema de escoamento comum, que direcionava a água para um reservatório externo de $7 \mathrm{~L}$ de volume útil. Dentro desse reservatório, foi instalado um biofiltro com volume de $3 \mathrm{~L}$. A água tratada era enviada de volta para os tanques por um sistema de bombeamento. Uma tela de $1 \mathrm{~mm}$ de diâmetro foi instalada no sistema de escoamento de cada tanque para evitar o escape das larvas.

Os fluxos de água nos diferentes tratamentos foram: $1.200 \pm 360 \mathrm{~mL} \mathrm{~min}^{-1}$ no $\mathrm{CMe} ; 1.100 \pm 400 \mathrm{~mL} \mathrm{~min}^{-1}$ no CMa; $1.400 \pm 200 \mathrm{~mL} \mathrm{~min}^{-1}$ no CB; $1.300 \pm 340 \mathrm{~mL} \mathrm{~min}^{-1}$ no $\mathrm{SF}$ e $1.400 \pm 400 \mathrm{~mL} \mathrm{~min}^{-1}$ no SB.

Trinta dias antes do experimento, os substratos foram mantidos em uma caixa de polietileno de $500 \mathrm{~L}$ com água, sob forte aeração. Foram adicionadas, semanalmente, pequenas quantidades de ração com $55 \%$ de proteína bruta para a maturação e fixação das

Pesq. agropec. bras., Brasília, v.44, n.5, p.511-518, maio 2009 
bactérias nitrificantes. Análises das concentrações de íon amônio e nitrato foram realizadas, também semanalmente, para verificar a ocorrência do processo de nitrificação.

As larvas de pacamã foram alimentadas com náuplios de Artemia sp. (eclodidos em água salinizada a 30\%o), nas quantidades diárias previamente estabelecidas de 700, 1.050 e 1.400 náuplios de Artemia sp. por larva de pacamã do $1^{\circ}$ ao $5^{\circ}$, do $6^{\circ}$ ao $10^{\circ}$ e do $11^{\circ}$ ao $15^{\circ}$ dia de alimentação, respectivamente, divididos em três refeições diárias, às 9,13 e $17 \mathrm{~h}$.

Durante o experimento, às $8 \mathrm{~h}$, foram medidos a temperatura da água $\left(26,4 \pm 1,2^{\circ} \mathrm{C}\right)$ e o oxigênio dissolvido ( $>5 \mathrm{mg} \mathrm{L}^{-1}$ ) (usando o oxímetro YSI 55) e o $\mathrm{pH}$ (usando um medidor de $\mathrm{pH}$ portátil Quimis Q400H). A cada três dias, às $8 \mathrm{~h}$, foram coletadas amostras de água para a determinação da alcalinidade (método potenciométrico) e das concentrações de nitrito, nitrato, íon amônio (Koroleff, 1976) e ortofosfato (Golterman et al., 1978). Diariamente, após a coleta de água, foi feita a sifonagem para remoção dos dejetos dos tanques, e 10\% do volume de água foi trocado.

Para avaliação do crescimento, três animais de cada repetição foram coletados aos cinco e dez dias de alimentação, e 15 animais foram coletados ao final do trabalho, para biometria (peso e comprimento total). Aos 15 dias de alimentação, término do experimento, também foi determinada a sobrevivência, cujos valores percentuais foram transformados para arco seno. Os parâmetros de crescimento, $\mathrm{pH}$ e alcalinidade foram submetidos à análise de variância (ANOVA) e ao teste de Tukey. Os dados das concentrações de íon amônio, nitrito, nitrato e ortofostato foram submetidos à ANOVA Fatorial de duas vias. Foram comparados os tratamentos entre os diferentes dias de coleta e os valores dentro de cada tratamento ao longo do tempo. As médias foram comparadas pelo teste de Tukey. As análises foram realizadas pelo programa SigmaStat 3.1 (Systat Software, 2002).

\section{Resultados e Discussão}

A sobrevivência de larvas de L. alexandri foi menor $(\mathrm{p}<0,05)$ no sistema com biofiltro externo ao tanque $(51,8 \pm 3,7 \%)$. Ao contrário do presente trabalho, para a piracanjuba (Brycon orbignyanus), que apresenta natação ativa na coluna de água, o uso do sistema com circulação de água com biofiltro externo não afetou a sobrevivência quando comparado ao uso de pedra porosa, sem biofiltro (Pedreira, 2003). Essas diferenças podem estar relacionadas ao comportamento de natação e posição das espécies na coluna de água, uma vez que o $L$. alexandri é sedentário e tem preferência por ambientes lênticos (Travassos, 1959). Dessa forma, o fluxo de água gerado no sistema com biofiltro externo ao tanque pode ter afetado a sobrevivência de L. alexandri, pois larvas mortas foram encontradas na tela de saída dos tanques. Para os demais tratamentos foram registrados valores semelhantes $(p>0,05) \mathrm{com}$ médias de $69,7 \pm 9,4 \%$ (biofiltro interno ao tanque com substrato de cascalho menor); 73,8 $\pm 7,1 \%$ (biofiltro interno ao tanque com substrato de cascalho maior); $68,2 \pm 4,3 \%$ (biofiltro interno ao tanque com substrato de concha e brita) e $68,3 \pm 4,9 \%$ (tanques sem biofiltro). $\mathrm{O}$ uso de diferentes substratos também não afetou a sobrevivência de juvenis de tilápia (Oreochromis niloticus) (Rhida \& Cruz, 2001).

Aos 5, 10 e 15 dias de criação, o crescimento de larvas de L. alexandri não foi afetado pelos diferentes sistemas utilizados (Tabela 1). Resultados semelhantes foram registrados para larvas de B. orbignyanus criadas em tanques sem biofiltro e com biofiltro externo ao tanque (Pedreira, 2003), e para juvenis de O. niloticus com diferentes substratos, como prolipropileno e polietileno (Rhida \& Cruz, 2001), rolos de plástico, cachimbos de PVC e pás "scrub" (Al-Hafedh et al., 2003).

Tabela 1. Média \pm desvio-padrão de peso e comprimento total de larvas de pacamã (Lophiosilurus alexandri), nos diferentes tratamentos de biofiltros e substratos, ao longo do experimento.

\begin{tabular}{lccc}
\hline Tratamento $^{(1)}$ & \multicolumn{3}{c}{ Dias de alimentação } \\
\cline { 2 - 4 } & \multicolumn{3}{c}{ Peso (mg) } \\
\hline & \multicolumn{3}{c}{15} \\
CMe & $36,4 \pm 4,8$ & $87,3 \pm 13,1$ & $134,8 \pm 8,7$ \\
CMa & $35,0 \pm 4,6$ & $81,4 \pm 18,6$ & $132,0 \pm 7,4$ \\
CB & $33,7 \pm 3,2$ & $61,1 \pm 11,2$ & $121,2 \pm 25,0$ \\
SF & $32,3 \pm 6,2$ & $79,9 \pm 17,1$ & $131,1 \pm 12,2$ \\
SB & $38,4 \pm 4,9$ & $78,6 \pm 12,3$ & $143,7 \pm 16,4$ \\
\hline & \multicolumn{3}{c}{ Comprimento (mm) } \\
CMe & $16,4 \pm 0,8$ & $22,1 \pm 0,5$ & $24,9 \pm 0,7$ \\
CMa & $16,9 \pm 0,4$ & $21,8 \pm 2,0$ & $24,4 \pm 0,3$ \\
CB & $16,6 \pm 0,3$ & $20,6 \pm 1,2$ & $24,2 \pm 1,1$ \\
SF & $16,7 \pm 0,6$ & $21,6 \pm 1,4$ & $23,8 \pm 0,4$ \\
SB & $17,3 \pm 0,4$ & $21,2 \pm 1,1$ & $24,5 \pm 0,4$ \\
\hline
\end{tabular}

${ }^{(1)} \mathrm{CMe}$, biofiltro interno ao tanque com substrato de cascalho menor; $\mathrm{CMa}$, biofiltro interno ao tanque com substrato de cascalho maior; $\mathrm{CB}$, biofiltro interno ao tanque com substrato de concha e brita; SF, biofiltro externo ao tanque com substrato de concha e brita; SB, tanque sem biofiltro. 
$\mathrm{O}$ pH é um parâmetro a ser avaliado em sistemas de recirculação de água por interferir no processo de nitrificação (Al-Hafedh et al., 2003). O tratamento biofiltro interno ao tanque, com substrato de concha e brita, proporcionou valores superiores de $\mathrm{pH}(7,5 \pm 0,2)$ $(p<0,05)$. Os demais tratamentos apresentaram valores inferiores e semelhantes entre si ( $p>0,05)$, com médias de $6,9 \pm 0,3$ (biofiltro interno ao tanque com substrato de cascalho menor), 6,9 $\pm 0,4$ (biofiltro interno ao tanque com substrato de cascalho maior), 7,3 $\pm 0,2$ (biofiltro externo ao tanque) e 7,1 $\pm 0,2$ (tanques sem biofiltro). $\mathrm{O} \mathrm{pH}$ mais elevado no tratamento com biofiltro interno ao tanque, com substrato de concha e brita, pode estar relacionado à presença de concha calcária no substrato. As diferenças de $\mathrm{pH}$ entre esse sistema e o sistema com biofiltro externo ao tanque, ambos com concha no substrato, podem ser atribuídas à relação entre a quantidade de substrato e o volume de água, uma vez que o biofiltro externo recebeu água de cinco tanques. Apesar das diferenças no $\mathrm{pH}$, esses valores estão dentro da faixa ideal, entre 6,8 e 8,0, para o processo de nitrificação (Masser et al., 1995).

A alcalinidade foi superior $(\mathrm{p}<0,05)$ nos tratamentos com biofiltro interno com substrato de brita e concha e com biofiltro externo aos tanques, com valores de $52,9 \pm 7,5$ e $56,9 \pm 9,7 \mathrm{mg} \mathrm{L}^{-1}$ de $\mathrm{CaCO}_{3}$, respectivamente. No sistema sem biofiltro, os valores foram intermediários $\left(29,3 \pm 2,7 \mathrm{mg} \mathrm{L}^{-1}\right.$ de $\left.\mathrm{CaCO}_{3}\right)$. Valores inferiores $(\mathrm{p}<0,05)$ foram registrados para $\mathrm{o}$ biofiltro interno ao tanque com substrato de cascalho menor $\left(23,9 \pm 4,5 \mathrm{mg} \mathrm{L}^{-1}\right.$ de $\left.\mathrm{CaCO}_{3}\right)$ e com substrato de cascalho maior $\left(21,8 \pm 7,4 \mathrm{mg} \mathrm{L}^{-1} \mathrm{de} \mathrm{CaCO}_{3}\right)$. Esse fato pode ser devido à composição dos substratos, já que as características da água são dadas pelo sedimento percolante (Schäfer, 1985). Para os biofiltros interno e externo, o substrato composto de conchas pode ter incrementado a alcalinidade. Contrariamente, para o uso de cascalho menor ou maior, a sílica, componente do quartzo, pode apresentar-se como ácido monossílico (Todeschini, 2004), reagir com o carbonato e diminuir a alcalinidade. Embora os valores registrados com o uso exclusivo de cascalho estejam abaixo do recomendado como ideal para sistemas fechados $\left(50 \mathrm{mg} \mathrm{L}^{-1}\right.$ de $\left.\mathrm{CaCO}_{3}\right)(\mathrm{Al}-\mathrm{Hafedh}$ et al., 2003), não foram limitantes para os animais e para o processo de nitrificação.

Para as concentrações de íon amônio, nitrito, nitrato e ortofosfato, foi verificado efeito significativo dos tratamentos $(\mathrm{p}<0,001)$, dos dias de cultivo $(\mathrm{p}<0,001)$ e também interação significativa entre tratamentos $\mathrm{x}$ dia de cultivo $(\mathrm{p}<0,001)$.

Foram observadas concentrações crescentes e superiores de íon amônio para o tratamento sem biofiltro ao longo do experimento (Tabela 2). A maior

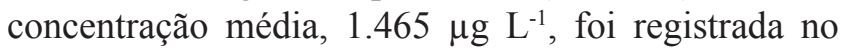
$13^{\circ}$ dia de cultivo. Para larvas e juvenis dessa espécie, a concentração letal de amônia não ionizada foi de 480 e $920 \mu \mathrm{g} \mathrm{L}^{-1}$, respectivamente, com o $\mathrm{pH}$ da água entre 8,2 e 8,5 em testes de 96 horas (Cardoso et al., 1996). No presente trabalho, como as larvas de pacamã apresentaram elevada sobrevivência e crescimento (Tabela 1), as concentrações de íon amônio registradas não afetaram os animais. A não toxicidade desse composto, no presente trabalho, pode estar relacionada aos menores valores de $\mathrm{pH}$, que influenciam diretamente o potencial tóxico da amônia (Schäfer, 1985), e à menor toxicidade do íon amônio.

Verificou-se, ainda, que o uso de biofiltro externo com substrato de concha e brita, biofiltro interno ao tanque com substrato de cascalho menor e biofiltro interno ao tanque com substrato de concha e brita apresentaram as menores concentrações de íon amônio, as quais permaneceram semelhantes entre si durante todo o experimento (Tabela 2). Para o uso do biofiltro interno com substrato de cascalho maior, foi

Tabela 2. Concentrações de íon amônio $\left(\mu \mathrm{g} \mathrm{L}^{-1}\right)$ durante a larvicultura de pacamã (Lophiosilurus alexandri), nos diferentes tratamentos de biofiltros e substratos, ao longo do experimento ${ }^{(1)}$.

\begin{tabular}{|c|c|c|c|c|c|c|}
\hline \multirow[t]{2}{*}{ Tratamento $^{(2)}$} & \multicolumn{6}{|c|}{ Dias de cultivo } \\
\hline & 1 & 4 & 7 & 10 & 13 & 16 \\
\hline$\overline{\mathrm{CMe}}$ & $33,0 \pm 12,2 \mathrm{Aa}$ & $199,1 \pm 197,1 \mathrm{Aa}$ & $142,5 \pm 161,0 \mathrm{Aa}$ & $279,4 \pm 452,6 \mathrm{Aa}$ & $33,5 \pm 12,0 \mathrm{Aa}$ & $32,4 \pm 15,8 \mathrm{Aa}$ \\
\hline $\mathrm{CMa}$ & $58,1 \pm 11,0 \mathrm{Aa}$ & $603,8 \pm 30,4 \mathrm{Bb}$ & $328,9 \pm 101,7 \mathrm{Aab}$ & $103,5 \pm 43,7 \mathrm{Aa}$ & $95,6 \pm 51,5 \mathrm{Aa}$ & $83,4 \pm 11,7 \mathrm{Aa}$ \\
\hline $\mathrm{CB}$ & $26,2 \pm 14,0 \mathrm{Aa}$ & $43,0 \pm 21,9 \mathrm{Aa}$ & $29,6 \pm 6,7 \mathrm{Aa}$ & $41,8 \pm 49,1 \mathrm{Aa}$ & $20,7 \pm 6,4 \mathrm{Aa}$ & $13,6 \pm 4,8 \mathrm{Aa}$ \\
\hline $\mathrm{SF}$ & $19,9 \pm 11,5 \mathrm{Aa}$ & $45,2 \pm 20,8 \mathrm{Aa}$ & $30,3 \pm 20,0 \mathrm{Aa}$ & $48,8 \pm 20,9 \mathrm{Aa}$ & $45,8 \pm 14,7 \mathrm{Aa}$ & $64,3 \pm 20,0 \mathrm{Aa}$ \\
\hline SB & $112,0 \pm 13,6 \mathrm{Aa}$ & $520,8 \pm 89,4 \mathrm{Bb}$ & $803,7 \pm 413,4 \mathrm{Bb}$ & $872,8 \pm 542,7 \mathrm{Bbc}$ & $1.465,5 \pm 355,8 \mathrm{Bd}$ & $1.149,3 \pm 80,0 \mathrm{Bcd}$ \\
\hline
\end{tabular}

${ }^{(1)}$ Médias \pm desvio-padrão seguidas de letras iguais, maiúsculas nas colunas e minúsculas nas linhas, não diferem entre si pelo teste de Tukey, a $5 \%$ de probabilidade. ${ }^{(2)} \mathrm{CMe}$, biofiltro interno ao tanque com substrato de cascalho menor; $\mathrm{CMa}$, biofiltro interno ao tanque com substrato de cascalho maior; $\mathrm{CB}$, biofiltro interno ao tanque com substrato de concha e brita; SF, biofiltro externo ao tanque com substrato de concha e brita; SB, tanque sem biofiltro. 
registrado aumento significativo nas concentrações de íon amônio nos primeiros dias, com redução no decorrer do experimento. Esse fato sugere um processo de nitrificação menos eficiente nesse tipo de substrato, que pode ser decorrente da menor superfície específica para a fixação das bactérias. Al-Hafedh et al. (2003) testaram diferentes tipos de substrato (rolos de plástico, cachimbos de PVC e pás "scrub") no cultivo de $O$. niloticus e verificaram que o uso de rolos de plástico foi mais eficiente na remoção de compostos nitrogenados devido à melhor passagem da água pelo substrato, que melhorou a eficiência de aproveitamento do oxigênio pelas bactérias.

Contrariamente ao presente trabalho, na larvicultura de sea bass (Dicentrarchus labrax), em sistema fechado de circulação de água, foi registrado incremento nas concentrações de amônia ao longo do cultivo, decorrente do fornecimento de Artemia sp. e do acúmulo de restos alimentares (Olívar et al., 2000). Para L. alexandri, apesar das quantidades crescentes de Artemia sp. fornecidas, as concentrações de íon amônio mantiveram-se constantes ao longo do tempo, o que confirma a eficiência dos sistemas de biofiltração utilizados. Esse fato sugere a sustentabilidade do sistema.
Para os sistemas com biofiltros externo e interno aos tanques com substrato de concha e brita, e para o uso de biofiltro interno ao tanque com substrato de cascalho menor, as concentrações de nitrito foram inferiores e semelhantes entre si durante o experimento (Tabela 3). Para o sistema de biofiltro interno ao tanque com substrato de cascalho maior, verificou-se aumento significativo nas concentrações de nitrito a partir do sétimo dia; as concentrações mantiveram-se constantes a partir do 13을 dia, o que confirma que nesse substrato o processo de nitrificação demora mais para iniciar. O sistema sem biofiltro apresentou concentrações de nitrito superiores aos demais tratamentos a partir do 13ํ dia de cultivo, com concentração média de $176 \mu \mathrm{g} \mathrm{L}^{-1}$ ao final do experimento.

Para o nitrato, as menores concentrações foram verificadas nos tanques sem biofiltro, a partir do sétimo dia de cultivo, e mantiveram-se inferiores aos demais tratamentos até o final do experimento (Tabela 4). Esse resultado sugere menor taxa de nitrificação. Porém, apesar da ausência de biofiltro, parte do íon amônio foi convertida em nitrato, o que indica nitrificação na coluna de água. Esse fato é corroborado por Ozório et al. (2004), que relatam a produção de nitrato em tanques sem biofiltro.

Tabela 3. Concentrações de nitrito $\left(\mu \mathrm{g} \mathrm{L}^{-1}\right)$ durante a larvicultura de pacamã (Lophiosilurus alexandri), nos diferentes tratamentos de biofiltros e substratos, ao longo do experimento ${ }^{(1)}$.

\begin{tabular}{|c|c|c|c|c|c|c|}
\hline \multirow[t]{2}{*}{ Tratamento $^{(2)}$} & \multicolumn{6}{|c|}{ Dias de cultivo } \\
\hline & 1 & 4 & 7 & 10 & 13 & 16 \\
\hline $\mathrm{CMe}$ & $0,4 \pm 0,1 \mathrm{Aa}$ & $0,4 \pm 0,1 \mathrm{Aa}$ & $12,2 \pm 16,8 \mathrm{Aa}$ & $10,1 \pm 8,0 \mathrm{Aa}$ & $3,0 \pm 1,3 \mathrm{Aa}$ & $2,7 \pm 0,9 \mathrm{Aa}$ \\
\hline $\mathrm{CMa}$ & $0,2 \pm 0,1 \mathrm{Aa}$ & $0,2 \pm 0,1 \mathrm{Aa}$ & $42,6 \pm 12,7 \mathrm{Bb}$ & $41,7 \pm 28,1 \mathrm{Bb}$ & $25,5 \pm 14,9 \mathrm{Aab}$ & $14,6 \pm 6,0 \mathrm{Aab}$ \\
\hline $\mathrm{CB}$ & $0,9 \pm 0,2 \mathrm{Aa}$ & $1,9 \pm 0,2 \mathrm{Aa}$ & $3,0 \pm 2,1 \mathrm{Aa}$ & $7,1 \pm 6,1 \mathrm{Aa}$ & $1,4 \pm 0,5 \mathrm{Aa}$ & $1,2 \pm 0,7 \mathrm{Aa}$ \\
\hline SF & $0,8 \pm 0,1 \mathrm{Aa}$ & $1,6 \pm 0,1 \mathrm{Aa}$ & $4,3 \pm 2,3 \mathrm{Aa}$ & $12,1 \pm 3,7 \mathrm{Aa}$ & $6,9 \pm 4,0 \mathrm{Aa}$ & $5,0 \pm 1,9 \mathrm{Aa}$ \\
\hline SB & $0,2 \pm 0,0 \mathrm{Aa}$ & $0,2 \pm 0,1 \mathrm{Aa}$ & $1,9 \pm 0,5 \mathrm{Aa}$ & $7,6 \pm 3,5 \mathrm{Aa}$ & $57,1 \pm 34,1 \mathrm{Bb}$ & $176,1 \pm 61,0 \mathrm{Bc}$ \\
\hline
\end{tabular}

${ }^{(1)}$ Médias \pm desvio-padrão seguidas de letras iguais, maiúsculas nas colunas e minúsculas nas linhas, não diferem entre si pelo teste de Tukey, a $5 \%$ de probabilidade. ${ }^{(2)} \mathrm{CMe}$, biofiltro interno ao tanque com substrato de cascalho menor; $\mathrm{CMa}$, biofiltro interno ao tanque com substrato de cascalho maior; $\mathrm{CB}$, biofiltro interno ao tanque com substrato de concha e brita; SF, biofiltro externo ao tanque com substrato de concha e brita; SB, tanque sem biofiltro.

Tabela 4. Concentrações de nitrato $\left(\mu \mathrm{g} \mathrm{L}^{-1}\right)$ durante a larvicultura de pacamã (Lophiosilurus alexandri), nos diferentes tratamentos de biofiltros e substratos, ao longo do experimento ${ }^{(1)}$.

\begin{tabular}{|c|c|c|c|c|c|c|}
\hline \multirow[t]{2}{*}{ Tratamento $^{(2)}$} & \multicolumn{6}{|c|}{ Dias de cultivo } \\
\hline & 1 & 4 & 7 & 10 & 13 & 16 \\
\hline$\overline{\mathrm{CMe}}$ & $100,0 \pm 10,6 \mathrm{Aa}$ & $399,6 \pm 83,5 \mathrm{ABb}$ & $7 \overline{57,2 \pm 87,2 \mathrm{Bb}}$ & $1.448,2 \pm 57,9 \mathrm{Bc}$ & $1.952,9 \pm 65,7 \mathrm{Bd}$ & $3.763,1 \pm 305,6 \mathrm{Be}$ \\
\hline $\mathrm{CMa}$ & $93,2 \pm 15,3 \mathrm{Aa}$ & $212,4 \pm 7,1 \mathrm{ABb}$ & $810,0 \pm 108,4 \mathrm{Bb}$ & $1.391,2 \pm 148,0 \mathrm{Bc}$ & $2.146,4 \pm 259,0 \mathrm{Bd}$ & $3.367,0 \pm 484,2 \mathrm{Be}$ \\
\hline $\mathrm{CB}$ & $122,6 \pm 10,4 \mathrm{Aa}$ & $659,6 \pm 117,2 \mathrm{Ba}$ & $820,7 \pm 66,1 \mathrm{Bb}$ & $1.474,4 \pm 76,0 \mathrm{Bc}$ & $2.316,2 \pm 56,4 \mathrm{Cd}$ & $4.713,7 \pm 804,7 \mathrm{Ce}$ \\
\hline SF & $177,1 \pm 12,8 \mathrm{Aa}$ & $676,2 \pm 38,9 \mathrm{Ba}$ & $1.013,9 \pm 90,9 \mathrm{Bb}$ & $1.571,0 \pm 10,3 \mathrm{Bc}$ & $2.419,2 \pm 77,3 \mathrm{Cd}$ & $4.607,3 \pm 294,3 \mathrm{Ce}$ \\
\hline SB & $88,8 \pm 14,4 \mathrm{Aa}$ & $100,2 \pm 8,5 \mathrm{Aa}$ & $102,8 \pm 12,2 \mathrm{Aa}$ & $263,5 \pm 84,6 \mathrm{Aa}$ & $622,3 \pm 302,0 \mathrm{Ab}$ & $2.451,3 \pm 998,9 \mathrm{Ac}$ \\
\hline
\end{tabular}

${ }^{(1)}$ Médias \pm desvio-padrão seguidas de letras iguais, maiúsculas nas colunas e minúsculas nas linhas, não diferem entre si pelo teste de Tukey, a $5 \%$ de probabilidade. ${ }^{(2)} \mathrm{CMe}$, biofiltro interno ao tanque com substrato de cascalho menor; $\mathrm{CMa}$, biofiltro interno ao tanque com substrato de cascalho maior; CB, biofiltro interno ao tanque com substrato de concha e brita; SF, biofiltro externo ao tanque com substrato de concha e brita; SB, tanque sem biofiltro. 
No quarto, sétimo e décimo dia de cultivo, os demais tratamentos apresentaram concentrações de nitrato semelhantes entre si. Porém, o uso de biofiltros externo e interno aos tanques com substrato de concha e brita mostrou uma melhor nitrificação dos produtos nitrogenados e apresentou as maiores concentrações de nitrato. O uso de biofiltro interno aos tanques com substrato de cascalho maior e menor apresentou valores intermediários. Para os diferentes sistemas de biofiltração, também foi registrado um aumento significativo nas concentrações de nitrato ao longo do experimento. Apesar da baixa toxicidade do nitrato para espécies de água doce (Tomasso, 1994), as concentrações de oxigênio no biofiltro devem ser mantidas adequadas. De acordo com van Rijn et al. (2006), em condições de anóxia pode ocorrer a desnitrificação, em que o nitrato é reduzido a amônia.

Segundo Colt et al. (2006), os diversos sistemas de biofiltração utilizados em aquicultura vêm apresentando diferentes desempenhos, o que dificulta comparações e torna árdua a seleção do melhor sistema a ser utilizado. Por exemplo, Greiner \& Timmons (1998) testaram dois substratos com diferentes diâmetros para o cultivo de tilápia (O. niloticus x aureus) e verificaram que o substrato de maior diâmetro tem uma taxa de nitrificação da amônia 7,5 vezes superior à do de menor diâmetro; contudo, o de menor diâmetro removeu do sistema aproximadamente 3,2 vezes mais amônia total por dia por unidade de volume. Lekang \& Kleppe (2000) utilizaram cascalho de diferentes tamanhos (2-4, 2-7 e 4-10 mm) e substratos comerciais e verificaram que, devido a uma maior área superficial, a melhor nitrificação ocorreu nos diferentes cascalhos. Contrariamente, Saliling et al. (2007) utilizaram diferentes tipos de substratos e verificaram eficiência semelhante entre eles. Na larvicultura de B. orbignyanus, o sistema fechado de circulação de água com biofiltro externo apresentou as menores concentrações de amônia e nitrito, enquanto para o nitrato foram observados valores semelhantes aos do sistema sem biofiltro (Pedreira, 2003). Asano et al. (2003) testaram biofiltro não submerso externo ao tanque e biofiltro submerso interno ao tanque para carpa-comum (Cyprinus carpio). Os autores registraram pior nitrificação no biofiltro interno, devido à maior exigência de oxigênio nesse tipo de biofiltro.

As maiores concentrações de ortofosfato (Tabela 5) foram registradas a partir do quarto dia para o sistema de biofiltro externo com substrato de concha e brita. $\mathrm{O}$ uso do biofiltro interno com substrato de concha e brita apresentou concentrações intermediárias no quarto, sétimo e décimo dia de cultivo, enquanto os demais tratamentos apresentaram concentrações inferiores nesses períodos. No $13^{\circ}$ e $16^{\circ}$ dia de cultivo, à exceção do sistema com biofiltro externo, todos os tratamentos apresentaram valores inferiores e semelhantes entre si. Ao analisar os diferentes tratamentos ao longo do tempo, verificou-se, de maneira geral, um acúmulo de ortofostato em todos os sistemas testados, com maiores concentrações no $13^{\circ}$ e $16^{\circ}$ dia de cultivo. Por este composto ser um dos principais responsáveis pela produtividade de fitoplâncton (Esteves, 1988), a sua remoção é desejável. Dessa forma, devem ser avaliados equipamentos e técnicas que possam reduzir as concentrações de ortofosfatos quando aliados ao sistema de biofiltração, diminuindo a possibilidade de eutrofização do ambiente.

Tabela 5. Concentrações de ortofosfato $\left(\mu \mathrm{g} \mathrm{L}^{-1}\right)$ durante a larvicultura de pacamã (Lophiosilurus alexandri), nos diferentes tratamentos de biofiltros e substratos, ao longo do experimento ${ }^{(1)}$.

\begin{tabular}{|c|c|c|c|c|c|c|}
\hline \multirow[t]{2}{*}{ Tratamento $^{(2)}$} & \multicolumn{6}{|c|}{ Dias de cultivo } \\
\hline & 1 & 4 & 7 & 10 & 13 & 16 \\
\hline $\mathrm{CMe}$ & $26,1 \pm 13,5 \mathrm{Aa}$ & $100,1 \pm 13,8 \mathrm{ABCb}$ & $134,3 \pm 25,4 \mathrm{Bbc}$ & $123,3 \pm 32,8 \mathrm{Bb}$ & $190,7 \pm 43,1 \mathrm{Bdc}$ & $194,1 \pm 49,2 \mathrm{Bd}$ \\
\hline $\mathrm{CMa}$ & $12,3 \pm 2,2 \mathrm{Aa}$ & $67,7 \pm 11,6 \mathrm{BCb}$ & $89,9 \pm 46,8 \mathrm{Bb}$ & $99,2 \pm 30,7 \mathrm{Bb}$ & $172,5 \pm 37,2 \mathrm{Bc}$ & $183,6 \pm 35,7 \mathrm{Bc}$ \\
\hline $\mathrm{CB}$ & $37,0 \pm 5,2 \mathrm{Aa}$ & $99,3 \pm 13,7 \mathrm{ABab}$ & $140,1 \pm 7,1 \mathrm{ABb}$ & $139,3 \pm 39,8 \mathrm{ABb}$ & $221,8 \pm 53,6 \mathrm{Bbc}$ & $251,3 \pm 73,2 \mathrm{Bc}$ \\
\hline $\mathrm{SF}$ & $37,7 \pm 2,4 \mathrm{Aa}$ & $126,4 \pm 0,7 \mathrm{Ab}$ & $196,4 \pm 1,7 \mathrm{Ac}$ & $197,2 \pm 8,8 \mathrm{Ac}$ & $297,8 \pm 4,2 \mathrm{Ad}$ & $334,5 \pm 2,2 \mathrm{Ad}$ \\
\hline$\underline{\text { SB }}$ & $18,1 \pm 1,9 \mathrm{Aa}$ & $64,6 \pm 12,7 \mathrm{Cab}$ & $106,8 \pm 17,8 \mathrm{Bb}$ & $124,9 \pm 56,7 \mathrm{Bb}$ & $215,9 \pm 82,2 \mathrm{Bc}$ & $188,7 \pm 39,6 \mathrm{Bc}$ \\
\hline
\end{tabular}

Pesq. agropec. bras., Brasília, v.44, n.5, p.511-518, maio 2009 


\section{Conclusões}

1. Os biofiltros melhoram as concentrações de compostos nitrogenados na água em função do tipo de substrato.

2. Os diferentes biofiltros e substratos não afetam o crescimento de larvas de pacamã (Lophiosilurus alexandri), porém, sua sobrevivência é menor no sistema de biofiltro externo ao tanque com substrato de brita e concha.

\section{Agradecimentos}

À Fundação de Amparo à Pesquisa do Estado de Minas Gerais, pelo apoio financeiro e pela concessão de bolsa; à Companhia de Desenvolvimento dos Vales do São Francisco e do Parnaíba, à Companhia Energética de Minas Gerais, ao Conselho Nacional de Desenvolvimento Científico e Tecnológico e ao Dr. Yoshimi Sato, pelo suporte para a realização deste trabalho.

\section{Referências}

AL-HAFEDH, Y.S.; ALAM, A.; ALAM, M.A. Performance of plastic biofilter media with different configuration in a water recirculation system for the culture of Nile tilapia (Oreochromis niloticus). Aquacultural Engineering, v.29, p.139-154, 2003.

ASANO, L.; AKO, H.; SHIMIZU, E.; TAMARU, C. Limited water exchange production systems for freshwater ornamental fish. Aquaculture Research, v.34, p.937-941, 2003.

CARDOSO, E.L.; CHIARINI-GARCIA, H.; FERREIRA, R.M.A.; POLI, C.R. Morphological changes in the gills of Lophiosilurus alexandri exposed to un-ionized ammonia. Journal of Fish Biology, v.49, p.778-787, 1996.

CHEN, S.L.; LING, J.; BLANCHETON, J.P. Nitrification kinetics of biofilm as affected by water quality factors. Aquacultural Engineering, v.34, p.179-197, 2006.

COLT, J.; LAMOUREUX, J.; PATTERSON, R.; ROGERS, G. Reporting standards for biofilter performance studies. Aquacultural Engineering, v.34, p.377-388, 2006.

ESTEVES, F. de A. Fundamentos de limnologia. Rio de Janeiro: Interciência: Finep, 1988. 575p.

FERNANDES, R.; GOMES, L.C.; AGOSTINHO, A.A. Pesque-pague: negócio ou fonte de dispersão de espécies exóticas? Acta Scientiarum. Biological Sciences, v.25, p.115-120, 2003.

GOLTERMAN, H.L.; CLYMO, R.S.; OHNSTAD, M.A.M. Methods for physical and chemical analysis of freshwaters. $2^{\text {nd }}$ ed. Oxford: Blackwell Scientific Publications, 1978. (IPB Handbook, 8).
GREINER, A.D.; TIMMONS, M.B. Evaluation of the nitrification rates of microbead and trickling filters in an intensive recirculating tilapia production facility. Aquacultural Engineering, v.18, p.189-200, 1998.

GUTIERREZ-WING, M.T.; MALONE, R.F. Biological filters in aquaculture: trends and research directions for freshwater and marine applications. Aquacultural Engineering, v.34, p.163-171, 2006.

HAGOPIAN, D.S.; RILEY, J.G. A closer look at the bacteriology of nitrification. Aquacultural Engineering, v.18, p.223-244, 1998.

HUSSAR, G.J.; CONCEIÇÃO, C.H.Z. da; PARADELA, A.L.; BARIN, D.J.; JONAS, T.C.; SERRA, W.; GOMES, J.P.R. Uso de leitos cultivados de vazão subsuperficial na remoção de macronutrientes de efluentes de tanques de piscicultura. Engenharia Ambiental, v.1, p.25-34, 2004.

KOROLEFF, F. Determination of nutrients. In: GRASSHOFF, K. (Ed.). Methods of sea water analysis. Weinheim: Verlag Chemie, 1976. p.117-181.

KUBITZA, F. Qualidade da água na produção de peixes. Parte II. Revista Panorama da Aqüicultura, v.8, p.35-41, 1998.

LEKANG, O.I.; KLEPPE, H. Efficiency of nitrification in trickling filters using different filter media. Aquacultural Engineering, v.21, p.181-199, 2000.

LINS, L.V.; MACHADO, A.B.M.; COSTA, C.M.R.; HERRMANN, G. Roteiro metodológico para elaboração de listas de espécies ameaçadas de extinção. Belo Horizonte: Fundação Biodiversitas, 1997. v.1. 50p. (Fundação Biodiversitas. Publicações avulsas, 1).

LÓPEZ, C.M.; SAMPAIO, E.V. Sobrevivência e crescimento larval do pacamã Lophiosilurus alexandri Steindachner, 1876 (Siluriformes, Pimelodidae), em função de três densidades de estocagem em laboratório. Acta Scientiarum. Biological Sciences, v.22, p.491-494, 2000.

LUZ, R.K.; SANTOS, J.C.E. dos. Densidade de estocagem e salinidade da água na larvicultura do pacamã. Pesquisa Agropecuária Brasileira, v.43, p.903-909, 2008.

LYSSENKO, C.; WHEATON, F. Impact of positive ramp shortterm operating disturbances on ammonia removal by trickling and submerged-upflow biofilters for intensive recirculating aquaculture. Aquaculture Engineering, v.35, p.26-37, 2006.

MASSER, M.P.; RAKOCY, J.; LOSORDO, T.M. Recirculating aquaculture tank production systems: management of recirculation systems. Louisiana: Louisiana State University Agriculture Center: Louisiana Cooperative Extension Service, 1995. p.1-12.

MAXIMIANO, A. de A.; FERNANDES, R.O. de; NUNES, F.P.; ASSIS, M.P. de; MATOS, R.V. de; BARBOSA, C.G.S.; OLIVEIRA-FILHO, E.C. Use of veterinary drugs and pesticides in the aquatic environment: demands, regulation and concerns on risks to human and environmental health. Ciência e Saúde Coletiva, v.10, p.481-491, 2005.

OLÍVAR, M.P.; AMBROSIO, P.P.; CATALÁN, I.A. A closed water recirculation system for ecological studies in marine fish larvae: growth and survival of sea bass larvae fed with live prey. Aquatic Living Resource, v.13, p.29-35, 2000. 
OZÓRIO, R.O.A.; AVNIMELECH, Y.; CASTAGNOLLI, N. Sistemas intensivos fechados de produção de peixes. In: CYRINO, J.E.P.; URBINATI, E.C.; FRACALOSSI, D.M.; CASTAGNOLLI, N. (Ed.). Tópicos especiais em piscicultura de água doce tropical intensiva. São Paulo: TecArt, 2004. p.7-24.

PEDREIRA, M.M. Comparação entre três sistemas no cultivo de larvas de piracanjuba (Brycon orbignyanus). Revista Ceres, v.50, p.779-786, 2003.

PEDREIRA, M.M.; SANTOS, J.C.E. dos; SAMPAIO, E.V.; FERREIRA, F.N.; SILVA, J. de L. Efeito do tamanho da presa e do acréscimo de ração na larvicultura de pacamã. Revista Brasileira de Zootecnia, v.37, p.1144-1150, 2008.

RHIDA, M.T.; CRUZ, E.M. Effect of biofilter media on water quality and biological performance of the Nile Tilapia (Oreochromis niloticus L.) reared in a simple recirculating system. Aquacultural Engineering, 24, p.157-166, 2001.

SALILING, W.J.B.; WESTERMAN, P.W.; LOSORDO, T.M. Wood chips and wheat straw as alternative biofilter media for denitrification reactors treating aquaculture and other wastewaters with high nitrate concentrations. Aquacultural Engineering, v.37, p.222-237, 2007.

SANTOS, J.C.E. dos; LUZ, R.K. Effect of salinity and prey concentrations on Pseudoplatystoma corruscans, Prochilodus costatus and Lophiosilurus alexandri larviculture. Aquaculture, v.287, p.324-328, 2009.

SATO, Y.; FENRICH-VERANI, N.; NUÑER, A.P.O.; GODINHO, H.P.; VERANI, J.R. Padrões reprodutivos de peixes da bacia do São Francisco. In: GODINHO, H.P.; GODINHO, A.L. (Ed.). Águas e peixes e pescadores do São Francisco das Minas Gerais. Belo Horizonte: PUC Minas, 2003. p.229-274.
SCHÄFER, A. Fundamentos de ecologia e biologia das águas continentais. Porto Alegre: Universidade Federal do Rio Grande do Sul, 1985. 532p.

SHIBATA, O.A. Family Pseudopimelodidae. In: REIS, R.E.; KULLANDER, S.O.; FERRARIS JUNIOR, C.J. Check list of the freshwater fishes of South and Central America. Porto Alegre: EDIPUCRS, 2003. p.401-405.

SYSTAT SOFTWARE. SigmaStat. Versión 3.1 for Windows. San Jose: Systat Software, 2002.

TENÓRIO, R.A.; SANTOS, A.J.G.; LOPES, J.P.; NOGUEIRA, E.M. de S. Crescimento do niquim (Lophiosilurus alexandri Steindachner 1876), em diferentes condições de luminosidade e tipos de alimentos. Acta Scientiarum. Biological Sciences, v.28, p.305-309, 2006.

TODESCHINI, M.L. Dinâmica espacial e temporal das características físicas e químicas do Rio Cubatão e distribuição espacial da Bacia Hidrográfica - litoral do Paraná. 2004. 124p. Dissertação (Mestrado) - Universidade Federal do Paraná, Curitiba.

TOMASSO, J.R. Toxicity of nitrogenous wastes to aquaculture animals. Reviews in Fish Science, v.2, p.291-314, 1994.

TRAVASSOS, H. Nótula sobre o pacamã Lophiosilurus alexandri Steindachner, 1876. Atas da Sociedade de Biologia, v.4, p.1-2, 1959.

TUNDISI, J.G. Água no século XXI: enfrentando a escassez. 2.ed. São Carlos: RiMa: IIE, 2003. 251p.

VAN RIJN, J.; TAL, Y.; SCHREIER, H.J. Denitrification in recirculating systems: theory and applications. Aquacultural Engineering, v.34, p.364-376, 2006.

Recebido em 16 de setembro de 2008 e aprovado em 30 de abril de 2009 